\title{
A pattern-filtering method for the determination of ocean bottom pressure anomalies from GRACE solutions
}

\author{
C. Böning, ${ }^{1}$ R. Timmermann, ${ }^{1}$ A. Macrander, ${ }^{1}$ and J. Schröter ${ }^{1}$ \\ Received 16 June 2008; revised 8 August 2008; accepted 20 August 2008; published 26 September 2008.
}

[1] Comparison of mass anomalies from the Gravity Recovery and Climate Experiment (GRACE) to in-situ ocean bottom pressure (OBP) data is complicated by the fact that GRACE estimates are representative for phenomena with scales of hundreds of $\mathrm{km}$, while OBP measurements are pointwise by nature. Simulations with the Finite Element Sea-ice Ocean Model (FESOM) indicate that on a monthly time scale OBP anomalies are coherent over large areas of complex geometry. A new filtering method for GRACE-derived ocean mass anomalies is obtained by applying these coherence patterns on a regular grid. Time series from OBP recorders all over the world ocean are used for validation. Compared to Gaussian filtering, the patternfiltering method improves the correlation between GRACE retrievals and in-situ OBP data (max. correlation now 0.87). Land leakage effects are eliminated almost completely. Finer scales can be resolved such that highly variable currents like the Gulf Stream can now be identified. Citation: Böning, C., R. Timmermann, A. Macrander, and J. Schröter (2008), A pattern-filtering method for the determination of ocean bottom pressure anomalies from GRACE solutions, Geophys. Res. Lett., 35, L18611, doi:10.1029/2008GL034974.

\section{Introduction}

[2] The GRACE satellite mission, launched in 2002, provides estimates of the variability of Earth's gravity field. Monthly solutions for gravity anomalies over the continents and the ocean represent mass variations of different origin and very different size. The land signal is dominated by the hydrological cycle in river basins [Ramillien et al., 2004] and snow accumulation in high latitudes [Frappart et al., 2006], the ocean signal by the redistribution of water due to circulation variability and to the seasonal cycles of river runoff and net evaporation. Retrieval of the small amplitudes of mass variations in the ocean, compared to the large hydrological signals over land, require a sophisticated filtering of GRACE data.

[3] In the open ocean, the different accuracies and spatial scales of satellite data and point measurements complicate comparisons between these two datasets. While GRACE estimates are representative for phenomena with a horizontal scale of several hundreds of $\mathrm{km}$, in situ ocean bottom pressure (OBP) measurements are pointwise by nature. Furthermore, spurious meridional patterns are evident in many GRACE satellite products. Therefore, the application

\footnotetext{
${ }^{1}$ Alfred Wegener Institute for Polar and Marine Research, Bremerhaven, Germany.
}

Copyright 2008 by the American Geophysical Union. 0094-8276/08/2008GL03497 of an adequate filter is necessary to allow for a comparison to in situ OBP data.

[4] A first approach is the application of a two dimensional Gauss function with a carefully chosen radius [Wahr et al., 1998]. However, due to the indiscriminative nature of the Gauss function, this method mixes land and ocean signals near the coasts. Furthermore, ocean circulation and thus OBP variations are strongly affected by bottom topography. Negative and positive OBP anomalies are often separated by topographic features like submarine ridges. An isotropic filter like the Gauss filter is bound to mix the signals of such a dipole.

[5] Swenson and Wahr [2006] developed a filtering mechanism which is based on the removal of correlated errors in the GRACE spherical harmonic solutions. Chambers [2006] improved this technique to study gravity variations over the ocean using an empirical approach to obtain an optimized set of parameters. This kind of filtering gives reasonable results on a global and ocean basin scale [Ponte et al., 2007], but also yields the risk of attenuation of signals. A comparison to point measurements or the determination of smaller-scale signals in general is therefore difficult.

[6] Using an equivalent Gauss filter radius of about $800 \mathrm{~km}$, Rietbroek et al. [2006] found a strong correlation between GRACE anomalies and OBP measurements in the Kerguelen and Crozet region. Their success already indicates that signals from an ocean bottom pressure recorder (BPR) represent large-scale ocean variability in this area. Thus, we expect OBP anomalies to be coherent over an area larger than the immediate vicinity of a BPR.

[7] This paper presents a newly developed GRACE data filtering algorithm which is based on the determination of an area of coherent signals around any given point. We will demonstrate that these patterns vary in size and can have a rather complex structure which partly follows bottom topography. For this purpose, we use results from a finite-element coupled sea ice - ocean model.

\section{Model and Data}

\subsection{Finite Element Sea Ice Ocean Model}

[8] To obtain information about large-scale ocean circulation, its variability, and its relation to OBP anomalies, we use the Finite Element Sea Ice - Ocean Model (FESOM) (R. Timmermann et al., On the representation of high latitudes in a finite-element global sea ice-ocean model, submitted to Ocean Modelling, 2008). The ocean module is a hydrostatic primitive-equation model which uses isopycnic diffusion and the Pacanowski and Philander [1981] vertical mixing scheme. The sea-ice component is a dynamic-thermodynamic sea-ice model with a prognostic snow layer using an elastic-viscous-plastic rheology. 
[9] Atmospheric sea-level pressure variations are considered in pressure computation throughout the water column. The ocean bottom pressure $p_{b}$ is computed as follows:

$$
p_{b}=\int_{0}^{\eta} \rho_{0} g d z+\int_{-H}^{0} \rho g d z+p_{a t}
$$

where $H$ represents the ocean depth, $\eta$ the sea surface height, $\rho_{0}$ the reference density $\left(1027 \mathrm{~kg} / \mathrm{m}^{3}\right), \rho$ the density, $g$ the acceleration due to gravity and $p_{a t}$ the atmospheric sea-level pressure. Computation of sea surface height variations includes contributions from net precipitation $P-E$, river runoff $R$, and the total water fluxes $F$ due the melting/freezing of ice and snow. To ensure the conservation of mass, we apply a steric correction for $\eta$ following Greatbatch [1994], so that

$$
\frac{\partial \eta}{\partial t}+d i v \int_{-H}^{\eta} \vec{u} d z+\int_{-H}^{\eta} \frac{1}{\rho} \frac{D \rho}{D t} d z=P-E+R+F,
$$

where $\vec{u}$ denotes the ocean velocity field.

[10] The model is run on a global grid with a horizontal resolution of $1.5^{\circ}$ and up to 26 z-levels. Atmospheric forcing data (daily datasets for $10-\mathrm{m}$ winds, $2-\mathrm{m}$ temperature, specific humidity, net precipitation, total cloudiness and sea-level pressure) are derived from the NCEP/NCAR reanalysis [Kalnay et al., 1996].

\subsection{GRACE Surface Mass Anomalies}

[11] We use mass anomaly fields derived from GRACE GFZ Release 04 [Schmidt et al., 2007]. The atmospheric and oceanic de-aliasing GAD products are added back to the GSM fields (for a detailed description see S. Bettadpur, Level-2 Gravity Field Product User Handbook, 2007, http://isdc.gfz-potsdam.de/index.php?name = UpDownloa$\mathrm{d} \&$ req $=$ getit?lid $=400)$. Solutions are expressed in spherical harmonic coefficients of degree and order 1 to 50. The degree 1 coefficients are obtained from the geocenter motion model of Swenson et al. [2008]. A gentle cut is applied to remove the influence of increasing errors for higher orders. The gentle cut $W_{l}$ is a 4 th order polynomial starting at degree 1 with point symmetry at degree 24. OBP anomalies $p_{G}{ }^{\prime}$ are derived from monthly means of November 2002 to January 2005 (with data gaps in January and June 2003, and January and August 2004). All time series $p_{G}$ were mapped to the global FESOM model grid as follows:

$$
\begin{gathered}
p_{G}=\frac{a g \rho_{E}}{3} \sum_{l=1}^{50} \sum_{m=0}^{l} \frac{2 l+1}{1+k_{l}} W_{l} \tilde{P}_{l m}(\sin \theta) \\
\cdot\left[C_{l m}^{\prime} \cos (m \Phi)+S_{l m}^{\prime} \sin (m \Phi)\right],
\end{gathered}
$$

where $a$ is the Earth's radius, $C_{l m}^{\prime}$ and $S_{l m}^{\prime}$ are the time dependent components of the GRACE spherical harmonic coefficients of degree $l$ and order $m, \tilde{P}_{l m}$ are the fully normalized associated Legendre functions, $k_{l}$ is the load Love number of degree $l, \rho_{E}$ is the Earth's average density, and $\mathrm{g}$ the gravitational acceleration. The anomalies $p_{G}^{\prime}$ are computed with a reference to the mean over the full period.

\subsection{Ocean Bottom Pressure Data}

[12] To assess the ability of GRACE to measure mass transport changes, a validation against in-situ OBP time series from different parts of the World Ocean is essential. In the framework of this project, all available OBP data that cover the GRACE operation period 2002-present have been collected to form an in-situ OBP database. We use time series from 66 deployments at 37 different locations, including data from the ASOF, Damocles, GRACE, MOVE, GLOUP, POL-ACCLAIM, NOAA-DART and NDBC projects (see auxiliary material ${ }^{1}$ ). All data have been quality controlled; outliers, pre-deployment values and pressure jumps have been removed.

[13] A common problem with pressure sensors is the long-term drift, with large trends during the first weeks to months of the deployment, and a smaller drift later. The nonlinear trend prohibits a linear interpolation between preand post-deployment calibrations (if these are available at all). Therefore, sensor drift was eliminated by an empirical exponential-linear least squares fit. Thus, these data cannot be used to evaluate annual to interannual OBP trends. On seasonal and shorter timescales, however, the accuracy of in-situ measurements is much better. The widely used pressure sensors from Paroscientific, for example, achieve a resolution of 0.001 dbar [University of Rhode Island, 2006]. Thus, short-term pressure anomalies of $1 \mathrm{~mm} \mathrm{SSH}$ change are detectable. For comparison with GRACE, monthly averages were calculated using the same time span as in the corresponding GRACE solutions.

\section{Pattern Filtering Method}

[14] We use FESOM simulations to identify patterns of coherent OBP variability. While modeled time series of monthly OBP anomalies from 1958 to 2005 were available, we only used data for the period 2002-2005 to be consistent with the start of the GRACE mission. To eliminate the seasonal cycle (which yields the dominant signal but on a much larger, hemispheric scale), a five months high-pass filter has been applied to the anomalies. From the resulting dataset $p_{F}^{\prime}$ we determine areas of high coherency by calculating the cross-correlation between the time series at fixed positions (positions of BPRs for the validation against in-situ measurements, model grid points for the global analysis) and time series at all points of the model domain within a $20^{\circ}$ radius. The correlation between an anomaly at a position $\vec{x}_{0}$ and one at a position $\vec{x}$ is defined as

$$
r\left(\vec{x}, \vec{x}_{0}\right)=\frac{E\left(p_{F}^{\prime}(\vec{x}) \cdot p_{F}^{\prime}\left(\vec{x}_{0}\right)\right)}{\sigma_{p_{F}^{\prime}(\vec{x}) \cdot \sigma_{p_{F}^{\prime}}^{\prime}\left(\vec{x}_{0}\right)}},
$$

where the $p_{F}^{\prime}$ are the high-pass filtered FESOM OBP anomalies, $E$ is the expected value and $\sigma$ is the standard deviation. The pattern of high coherency ("coherence pattern") is then defined as the area with correlations larger than 0.7 .

[15] It turns out that OBP anomalies on a time scale of 15 months are spatially coherent over large areas which are

\footnotetext{
${ }^{1}$ Auxiliary materials are available in the HTML. doi:10.1029/ 2008 GL034974.
} 

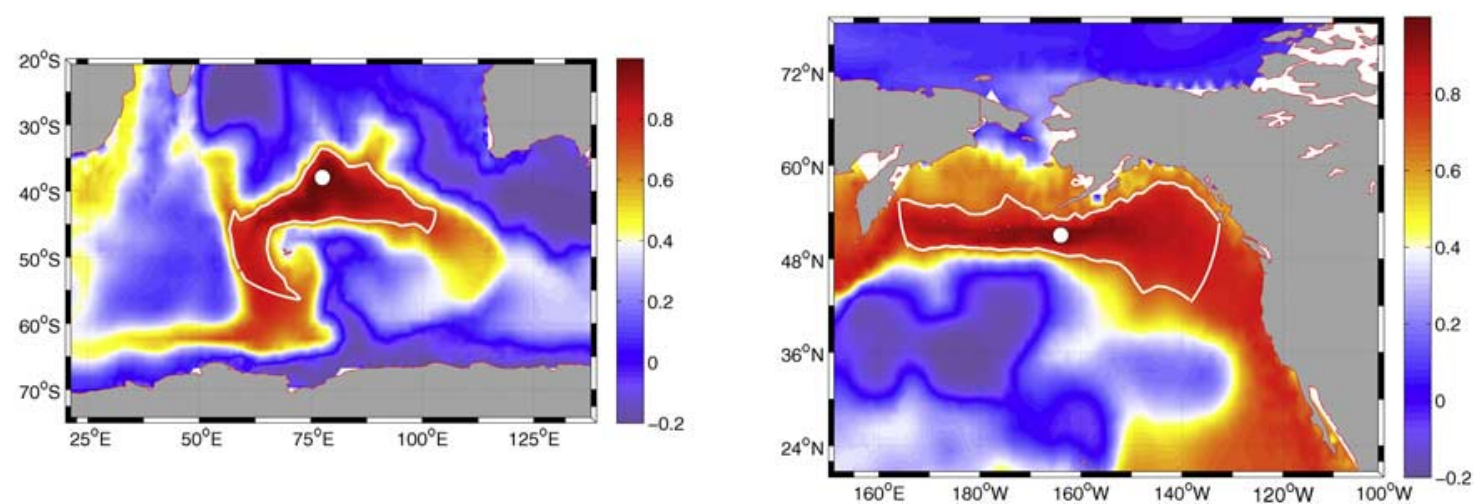

Figure 1. Two examples of model-derived patterns of high spatial coherency ("coherence patterns"), defined by the areas with correlations larger than 0.7 inside a circle with $20^{\circ}$ radius. Examples refer to (left) the BPRs close to Amsterdam Island and (right) the NOAA Dart Array. White dots indicate the reference points (BPR positions), white lines the cut-off edge.

strongly related to bottom topography. For a BPR position close to Amsterdam Island for example, we find a coherency pattern with a diameter of approximately $1500 \mathrm{~km}$, for a position in the NOAA/DART Array we find a pattern diameter of approximately $1000 \mathrm{~km}$ (Figure 1). OBP measurements at these positions are representative for the areas defined by the coherence patterns.

[16] We use these coherence patterns to filter the GRACE data by weighting the data for each position inside the $20^{\circ}$ circle with the pre-computed correlation coefficient. The weighting function $r^{*}$ is defined with a cut-off at a correlation of 0.7 as

$$
r^{*}\left(\vec{x}, \vec{x}_{0}\right)= \begin{cases}r,\left(\vec{x}, \vec{x}_{0}\right), & \text { for } r\left(\vec{x}, \vec{x}_{0}\right) \geq 0.7 \\ 0, & \text { for } r\left(\vec{x}, \vec{x}_{0}\right)<0.7\end{cases}
$$

[17] In order to generate a smooth transition in areas where the maximum distance criterion holds and the correlation is still above 0.7 , a monotonically decreasing cut-off function centered at a distance of $18^{\circ}$ is additionally applied:

$$
\zeta\left(\vec{x}, \vec{x}_{0}\right)=\frac{1-\tanh \left(\frac{\Delta\left(\vec{x}, \vec{x}_{0}\right)-18^{\circ}}{2^{\circ}}\right)}{2},
$$

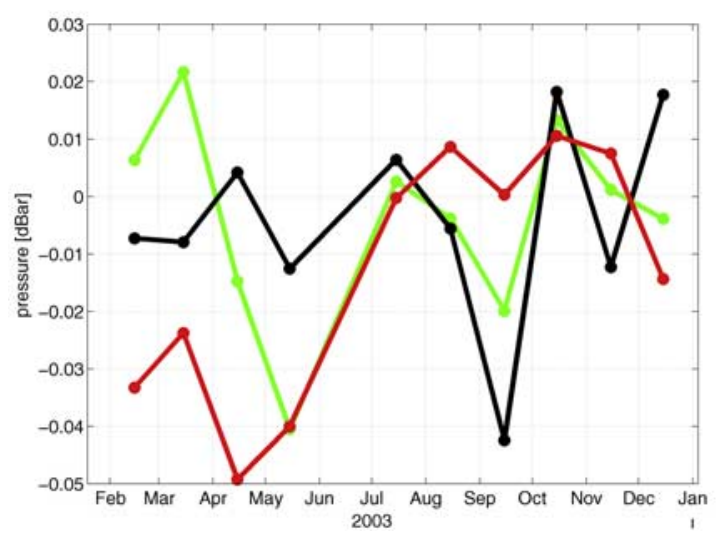

where $\Delta$ is the distance [in degrees] between $\vec{x}_{0}$ and $\vec{x}$. The filtered GRACE solution $p_{G}{ }^{*}\left(\vec{x}_{0}\right)$ is then calculated by

$$
p_{G}^{*}\left(\vec{x}_{0}\right)=\frac{\int_{C} p_{G}^{\prime}(\vec{x}) \cdot r^{*}\left(\vec{x}, \vec{x}_{0}\right) \cdot \zeta\left(\vec{x}, \vec{x}_{0}\right) d A}{\int_{C} r^{*}\left(\vec{x}, \vec{x}_{0}\right) \cdot \zeta\left(\vec{x}, \vec{x}_{0}\right) d A},
$$

where $C$ is a circle with a radius of $20^{\circ}$ around $\vec{x}_{0}$. The result for every $\vec{x}_{0}$ is a time series that is comparable to the point measurement of an OBP recorder. By applying this procedure to every point of the oceanic $1.5^{\circ} \times 1.5^{\circ}$ grid, we obtain maps of GRACE-derived mass anomalies.

\section{Results}

[18] As a reference dataset, we use GRACE data that have been smoothed with a $750 \mathrm{~km}$ Gauss filter. The radius has been determined from studies in which OBP anomalies derived from GRACE data were filtered with different Gauss radii and then compared to in situ data. It was found that a radius of $750 \mathrm{~km}$ yields the best results.

[19] Even with the optimized radius, a comparison with BPR data reveals that OBP anomalies derived from the GRACE solutions and filtered with a Gauss filter fail to

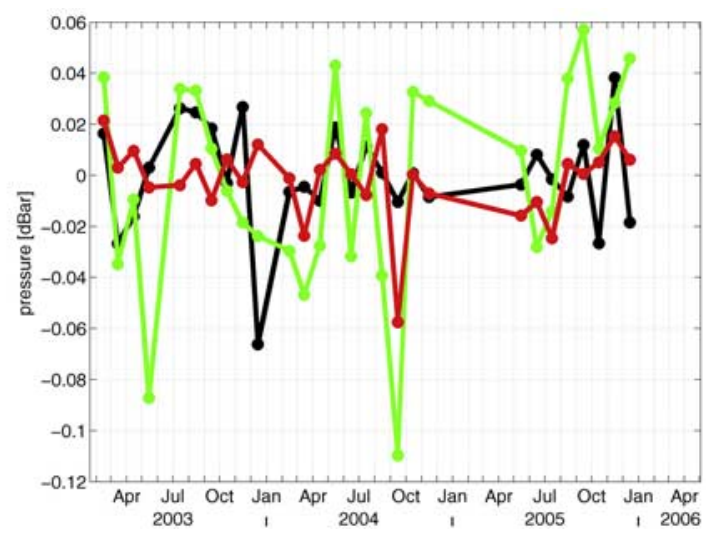

Figure 2. Time series of OBP anomalies at $77.58^{\circ} \mathrm{E}, 37.9^{\circ} \mathrm{S}\left(\mathrm{CNES}\right.$, Amsterdam Island) and $164^{\circ} \mathrm{W}$, $51.07^{\circ} \mathrm{N}$ (NOAA DART Array). Black line indicates in situ observations, red line the Gauss-filtered GFZ RL04, green line pattern-filtered GFZ RL04. 

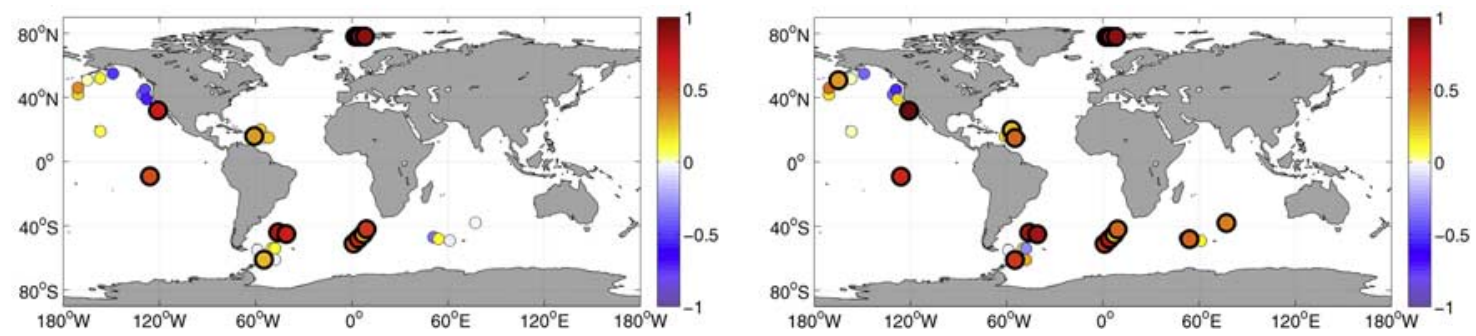

Figure 3. Correlations between in situ and GRACE-derived OBP anomalies. (left) Gauss-filtered GRACE data. (right) Pattern-filtered GRACE data. Large dots indicate a correlation that is significant at an $85 \%$ level.

reproduce the characteristic extremes of the in situ time series in many cases. Data filtered with the coherence pattern method feature a better agreement. Two typical examples are shown in Figure 2. Close to Amsterdam Island, the Gauss-filtered solution fails to reproduce the observations in amplitude and phase. The course of minima and maxima is much better captured in the pattern-filtered time series. Gauss-filtering in the NOAA Dart Array causes a slight underestimation of the amplitude for most of the time. The characteristic extremes are not well captured. The pattern-filtered solution features a higher amplitude and reproduces a larger part of the observed variability. Correlations between GRACE and in situ time series increase from -0.01 to 0.4 for the Amsterdam Island position and from 0.02 to 0.32 for the BPR in the NOAA Dart Array. For both positions, the correlation is only significant (at a level of at least $85 \%$ ) when the pattern filter is applied.

[20] Applying the pattern-filtering algorithm to GRACE GFZ RL04 monthly solutions for other BPR positions all over the globe yields an improved correlation with in situ OBP time series in comparison to the Gauss filtered data in many cases (Figure 3). Especially in the Crozet/Kerguelen/ Amsterdam Islands region and in the southwestern and tropical Atlantic the agreement between GRACE and in situ measurements is enhanced. The correlation increases by $0.1-0.2$ at these locations, and even switches from a correlation close to zero to a significant positive correlation in the Crozet/Kerguelen/Amsterdam Islands region. Applying a Gauss filter to the GRGS solution, Rietbroek et al. [2006] found an even higher correlation in the Crozet/ Kerguelen region; however, a comparison to other BPRs shows that the agreement strongly varies with location and is weaker than for the GFZ solution in other places. For 18 positions, the correlation between pattern-filtered GFZ data and in situ measurements is significant at the $85 \%$ level; for 7 of them the correlation is significant even at the $95 \%$ level. Compared to the Gauss-filtered data with only 14 (3) positions significant at the $85 \%$ (95\%) level, the improvement is obvious.

[21] Global maps of root-mean-square (RMS) monthly variability of the GRACE solutions filtered with a $750 \mathrm{~km}$ Gauss filter (Figure 4, left) show a high variability in and adjacent to the seasonally ice-covered areas. The ocean signal in coastal regions is highly influenced by the large hydrologic cycle over land due to the indiscriminative nature of the Gauss filter; the signature of land leakage effects is evident. In low and mid latitudes the signals are very smooth, but spurious meridional patterns are still visible. Due to the smoothness of the variability patterns it is difficult to identify circulation patterns or other oceanographic signals correlated to OBP anomalies.

[22] Monthly RMS variability derived from patternfiltered solutions (Figure 4, right) shows a similar structure with high variability in the high latitudes. However, the an-isotropy of the pattern-filtering does not smooth out local gradients and features on a much smaller scale can be clearly identified. Specifically, most of the currents with high variability are well pronounced. Western boundary currents like the Gulf Stream or the Kuroshio are as clearly visible as for example the Zapiola Eddy (a stationary quasi-barotropic anticyclonic eddy centered at $45^{\circ} \mathrm{S} 42^{\circ} \mathrm{W}$ east of South America) and the Agulhas Return Current. The clear representation and sharp boundaries of these features, especially the boundary currents, and the reduced variability along the coast indicate a good reduction of land leakage effects - which is particularly well visible close to Amazon Basin.

[23] Averaging the filtered data over the entire global ocean yields a seasonal cycle with a maximum in Sept./ Oct. and a minimum in April, which is very similar in the two datasets (not shown). While an amplitude of less than $0.5 \mathrm{~cm}$ equivalent water column height is found in the Gauss-filtered data, the pattern-filtered data feature an amplitude of about
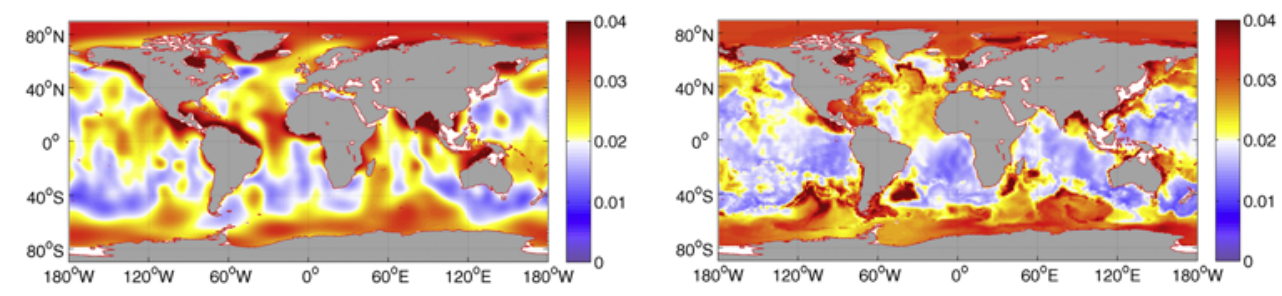

Figure 4. RMS monthly variability (in dbar) of GRACE GFZ RL04-derived OBP. (left) Gauss-filtered with $750 \mathrm{~km}$ radius. (right) Using the newly developed pattern-filtering algorithm. 
$0.7 \mathrm{~cm}$, which agrees well with results from Lombard et al. [2007].

[24] An analysis on the damping effects shows that the biases of the pattern filter and of the Gaussian filter are of the same order of magnitude. The gentle cut applied to the spherical harmonic coefficients reduces the noise but yields anomalies which still have a ten times higher amplitude than the in-situ data. When, in the second step, the pattern filter is applied, damping varies with regions due the different shapes of the patterns. On average, damping introduced by the pattern filtering matches that of a gauss filter with a radius of $750 \mathrm{~km}$.

\section{Conclusions}

[25] We have presented a newly developed patternfiltering method to obtain global fields of ocean mass anomalies from GRACE monthly solutions. The algorithm is based on the detection of patterns of coherent ocean bottom pressure variability; it uses information from modeled circulation patterns. We have demonstrated that the new method yields improvements for the correlation between GRACE-derived and in situ OBP anomalies. Not only is this true for the high latitudes where the Gauss-filtered solutions were already good at many locations, but also in the tropics and close to the coasts the pattern-filtered GRACE-derived OBP signals get closer to the observations, so that the number of positions with a significant correlation between GRACE and in situ data increases. Signatures of boundary currents are now very clear; land leakage effects are strongly reduced. The new pattern-filtering method thus provides the ability to extract features of oceanic water mass movements in more detail. It therefore promises to be an appropriate tool to use GRACE solutions to quantify ocean mass variations on a local as well as on a global scale.

[26] Acknowledgments. We would like to thank S. Klosko and an anonymous reviewer for their helpful comments and suggestions. The NCEP/NCAR reanalysis data were provided by the NOAA Climate Diagnostics Center, Boulder, online at http://www.cdc.noaa.gov. Some of the model experiments utilized resources from the North German Alliance for the Advancement of High-Performance Computing (HLRN). The German Ministry of Education and Research (BMBF) supports the GRACE project within the GEOTECHNOLOGIEN geoscientific R\&D programme under grant $03 \mathrm{~F} 0423 \mathrm{E}$.

\section{References}

Chambers, D. (2006), Evaluation of new GRACE time-variable gravity data over the ocean, Geophys. Res. Lett., 33, L17603, doi:10.1029/ 2006GL027296.

Frappart, F., G. Ramillien, S. Biancamaria, N. M. Mognard, and A. Cazenave (2006), Evolution of high-latitude snow mass derived from the GRACE gravimetry mission (2002-2004), Geophys. Res. Lett., 33, L02501, doi:10.1029/2005GL024778.

Greatbatch, R. (1994), A note on the representation of steric sea level in models that conserve volume rather than mass, J. Geophys. Res., 99, $767-771$.

Kalnay, E., et al. (1996), The NCEP/NCAR 40-year reanalysis project, Bull. Am. Meteorol. Soc., 77, 437-471.

Lombard, A., D. Garcia, G. Ramillien, A. Cazenave, R. Biancale, J. M. Lemoine, F. Flechtner, R. Schmidt, and M. Ishii (2007), Estimation of steric sea level variations from combined GRACE and Jason-1 data, Earth Planet. Sci. Lett., 254(1-2), 194-202.

Pacanowski, R., and S. Philander (1981), Parameterization of vertical mixing in numerical models of tropical oceans, J. Phys. Oceanogr., 11, $1443-1451$

Ponte, R. M., K. J. Quinn, C. Wunsch, and P. Heimbach (2007), A comparison of model and GRACE estimates of the large-scale seasonal cycle in ocean bottom pressure, Geophys. Res. Lett., 34, L09603, doi:10.1029/ 2007GL029599.

Ramillien, G., A. Cazenave, and O. Brunau (2004), Global time variations of hydrological signals from GRACE satellite gravimetry, Geophys. $J$. Int., 158, 813-826.

Rietbroek, R., P. LeGrand, B. Wouters, J.-M. Lemoine, G. Ramilien, and C. W. Hughes (2006), Comparison of in situ bottom pressure data with GRACE gravimetry in the Crozet-Kerguelen region, Geophys. Res. Lett., 33, L21601, doi:10.1029/2006GL027452.

Schmidt, R., U. Meyer, C. Dahle, F. Flechtner, and J. Kusche (2007), Monthly and weekly EIGEN-GRACE05S gravity field solutions for monitoring of mass variations in the Earth system, paper presented at 2nd Space for Hydrology Workshop, Eur. Space Agency, Geneva.

Swenson, S., and J. Wahr (2006), Post-processing removal of correlated errors in GRACE data, Geophys. Res. Lett., 33, L08402, doi:10.1029/ 2005GL025285

Swenson, S., D. P. Chambers, and J. M. Wahr (2008), Estimating geocenter variations from a combination of GRACE and ocean model output, J. Geophys. Res., 113, B08410, doi:10.1029/2007JB005338.

University of Rhode Island (2006), Inverted Echo Sounder User's Manual, revised, report, Univ. of R. I., Narragansett.

Wahr, J., M. Molenaar, and F. Bryan (1998), Time variability of Earth's gravity field: Hydrological and oceanic effects and their possible detection using GRACE, J. Geophys. Res., 103, 30,205-30,229.

C. Böning, A. Macrander, J. Schröter, and R. Timmermann, Alfred Wegener Institute for Polar and Marine Research, Bussestrasse 24, Bremerhaven D-27570, Germany. (carmen.boening@awi.de) 